\title{
Edge Detection and Level Set Active Contour Model for the Segmentation of Cavity Present in Dental X-Ray Images
}

\author{
Akanksha Kaushik \\ School of Engineering and \\ Technology \\ ITM University \\ Sector-23A, Gurgaon 122017, \\ Haryana
}

\author{
Prakash C Mathpal \\ School of Engineering and \\ Technology \\ ITM University \\ Sector-23A, Gurgaon 122017, \\ Haryana
}

\author{
Vandini Sharma \\ School of Engineering and \\ Technology \\ ITM University \\ Sector-23A, Gurgaon 122017, \\ Haryana
}

\begin{abstract}
In the field of medical science, one of the oldest and frequently used techniques, for detecting fractures/defects in human bones is X-Ray. This is worth to do research on segmentation of X-Ray images and inventing the new techniques for the same. In this paper we used X-Ray Dental Images and Segmentation is done using Level Set Active Contour.
\end{abstract}

\section{Keywords}

Edge detection, image segmentation, digital dental (dd) x-ray image, level set active contour (lsac).

\section{INTRODUCTION}

Medical imaging system has been widely used in various medical applications like orthopedic, ultra sound and many more. Medical imaging is a process used to capture the images of human body, for clinical purposes like diagnosis, anatomy, physiology etc. In its broadest sense; medical imaging can also be said as biological imaging. A number of imaging technologies such as X-ray radiography, magnetic resonance imaging, medical ultra-sonography(generally called as ultrasound), endoscopy, elastography, tactile imaging, thermography etc. that are used to detect the bone structures, organs, DNA structures, blood cells and tissues etc.

\begin{abstract}
Apart from imaging, other techniques for measuring and recording purpose such as electrocardiography, electroencephalography, magneto encephalography, records the data in the form of graphs or maps that contain information about measurement locations. These measuring techniques may not be designed to produce images but can be considered as forms of medical imaging.

Clinical imaging is generally equated to radiology which uses invisible light spectrum such as gamma rays (used in positron emission tomography), X-rays (used in orthopedics to detect bone structures), ultraviolet rays (used in lasers, photo chemotherapy and phototherapy for the treatment of cancer). Various researches have been done to identify the cavities present in the image and following terms are widely used for that purpose:

Segmentation is an important as well as crucial process in medical image processing. Image segmentation is a process of isolating an object or region of interest from an entire image. This is done for partitioning a digital image into multiple segments, say set of pixels. Segmentation is done to represent an image into more meaning manner and easier to analysis. The result of segmentation is a set of segments or pixels that collectively cover the entire image and can be characterized on image properties like color, intensity, texture etc.
\end{abstract}

Image segmentation is broadly categorized into two types:

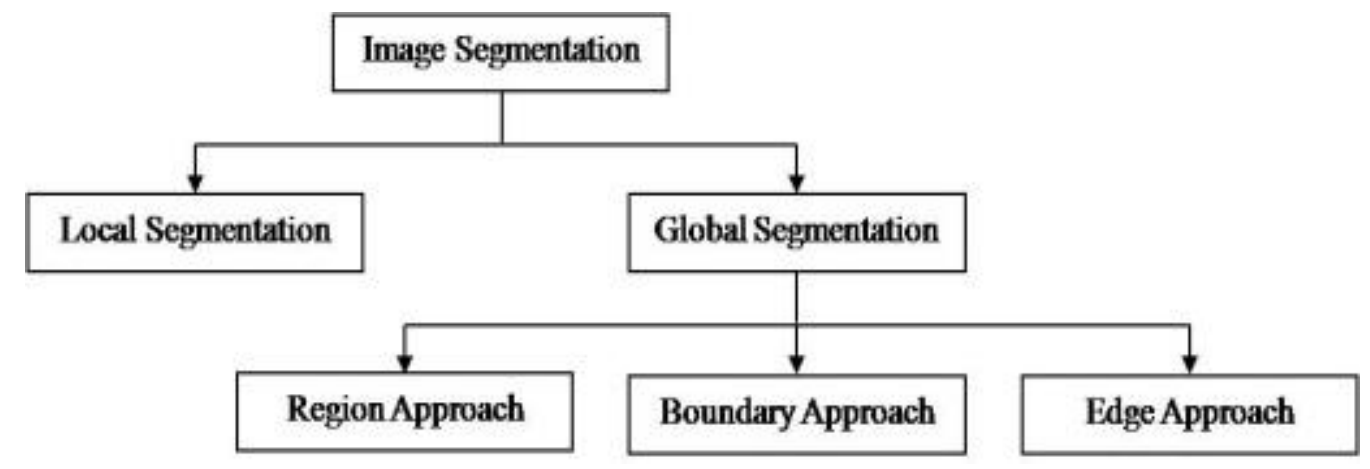

Figure 1: Categorization of Image Segmentation

Local segmentation In image segmentation, local segmentation is at the lowest level and operates only on small number of pixels. Local segmentation is widely used to achieve a number of image processing tasks such as image 
denoising, pixel classification, edge detection and pixel interpolation. Local segmentation is concerned with segmenting sub-image or the portions in the image. Local segmentation can be called as image enhancement process.

Global Segmentation Global segmentation is opposite of local segmentation. It is at highest level and deals with segmenting a whole image. In global segmentation, an image is partitioned into groups of pixels which are homogeneous with respect to some characteristics and each group is called as segment. Global segmentation is further categorized into three types: Region approach, Boundary approach and Edge approach. Clustering is one of the widely used technique in which clustering of pixels is done according to some homogeneity criteria. A clustering technique that is widely used is Fuzzy C- mean clustering. Balafar et al. [1] proposed new multi-scale medical image segmentation based on Fuzzy C-mean. In our paper, we have used edge approach i.e. Level Set Active Contour method for image segmentation.

\subsection{Methodology Used}

\subsubsection{Edge Detection}

Edge detection is a process of identifying the image in the form of lines spread over a particular object in an image. With the edge representation of an image, it becomes easy to perform further computations on the required region or object. There are a number of edge operators such as Roberts operator, sobel operator canny operator, preywitt operator etc. also, neural network can be used for detecting the edges over the object boundaries in a dental X-Ray image [2].

\subsubsection{Level Set Active Contour}

Image segmentation using active contour model is a very successful technique and is widely used in image processing. Active contour method was an earlier approach used for image segmentation. Active contour is also called as snake model. In snake model, an initial contour of snake like shape is set and further it dynamically adapts to the desired edges of the object in the image. But the disadvantage of snake model was that it does not work efficiently with the images having inhomogeneous intensities. Also, initial contour should be set near to the object to be segmented, so that the computation time for the contour to spread over the object boundaries should be less.

Advancement over snake model is level set method in which active contour models (snake model) are combined with geometric model. The level set method is a numerical technique that is used to track the interfaces and shapes. In level set method, the contours are set as zero level set of higher dimensional function, known as level set function. Initially, geometrical shaped contours are set and after a number of iteration the contours spread over the vision of interest or the edges of the object boundaries.

One of the papers [17], for segmentation suggested the following algorithm and got the promising results. In this paper, we are using the same algorithm for our experimental data and getting the results.

\subsection{Algorithm}

$\mathrm{N}=$ number of iterations,

For all $\mathrm{N}$ iterations

- From a point $\mathrm{x}$ on the image, set the boundary condition and find the shortest travelling time using distance regularization to the boundaries of the cavity.
- Estimate external energy and calculate PDE.

\section{- Energy Formulation}

Let $\Phi: \Omega \rightarrow \hat{\mathrm{R}}$, be an initial LSF defined on image domain $\Omega$. The Energy Function is defined by

$\mathrm{E}(\Phi)=\mu \mathrm{R}_{\mathrm{P}}(\Phi)+\mathrm{E}_{\text {ext }}(\Phi)$

where $R_{P}(\Phi)$ is the regularization term

$$
R_{P}(\Phi)=\int p(|\nabla| d x)
$$

here $\mathrm{p}$ is the potential function

\section{- Energy Minimization (Gradient Flow)}

To minimize the energy $\mathrm{E}(\Phi)$, a standard method is used i.e. the gradient flow equation

$$
\begin{aligned}
& \frac{\partial \Phi}{\partial t}=\frac{\partial F}{\partial \Phi} \\
& \text { where } \frac{\partial F}{\partial \Phi} \text { is the Gateaux derivative of the function } \\
& \mathrm{F}(\Phi)
\end{aligned}
$$

Gateaux derivative of the function $\mathrm{R}_{\mathrm{P}}(\Phi)$ is

$$
\frac{\partial R_{P}}{\partial \Phi}=\operatorname{div}\left(d_{p}(|\nabla \Phi|) \nabla \Phi\right)
$$

where div is the divergence operator The energy equation with Gateaux derivative can be written as

$$
\frac{\partial E}{\partial t}=\mu \frac{\partial R_{P}}{\partial \Phi}+\frac{\partial E_{e x t}}{\partial \Phi}
$$

The Gradient Flow of the energy $\mathrm{E}(\Phi)$ is

$$
\frac{\partial E}{\partial t}=-\mu \frac{\partial R_{P}}{\partial \Phi}-\frac{\partial E_{e x t}}{\partial \Phi}
$$

Putting the value of $\frac{\partial R_{P}}{\partial \Phi}$ in equation (6), we get

$$
\frac{\partial \Phi}{\partial t}=\mu \operatorname{div}\left(d_{p}(|\nabla \Phi|) \nabla \Phi\right)-\frac{\partial E_{e x t}}{\partial \Phi}
$$

This equation is the PDE or the level set evaluation equation. To solve the above PDE, use the boundary condition and initial function.

- $\quad$ Find the distance, curvature and speed at each point. Update Level Set Function for $\alpha=0$

$$
\text { End }
$$

End

\section{REVIEW OF LITERATURE}

Image segmentation is applied on images so as to isolate a region or object of interest from the entire image. There are number of image segmentation techniques that are classified into region approach, boundary approach and edge approach. We used edge based image segmentation techniques i.e. level set active contour for the segmentation of cavities in the image. Active contour method was an earlier edge based 
approach used for image segmentation. Active contour model is also called as snake model, first introduced by Kass [3] in 1987. Caselles et al. [4] presented a geodestic active contours scheme for the detection of object boundaries. Chenyang et al. [5] developed a new external force i.e. Gradient Vector Flow for solving the problem of initialization and poor convergence. Steve et al. [6] proposed a dual active contour method to relieve the problem of initialization of parameters and initial contour. Amelioration over active contour method was level set method, presented by Sathian [7] in 1999, in which geometric shapes are combined with the snake model. Giraldi et al. [8] presented a new approach, a combination of T-Snakes method and multi-resolution method for the segmentation and boundary extraction. Chan et al. [9] proposed a new method for the detection of objects, based on curve evolution and Mumford Shah function for the level set segmentation. Derraz et al. [10] presents Application of Active Contour Models in Medical Image Segmentation. Chunming et al. [11] presents a variational formulation for the level set evolution without re-initialization. Qin et al. [12] presented a New Medical Image Sequences Segmentation Based On Level Set Method. Chiu et al. [13] presents a method for the evaluation of Active contour on medical inhomogeneous image segmentation. Franz et al. [14] presented Fast and Robust Active Contours for Image Segmentation. Huang et al. [15] proposed a level set method for image segmentation in the presence of intensity inhomogenieties with application to MRI. Recently, Norouzi et al. [16] proposed a methodology for the segmentation and features extraction in digital dental X-Ray images.

\section{METHODOLOGY}

In this paper, we propose an identification of the defects/cavity present in the DD X-Ray images and an edge detection based segmentation of the cavity present in the image. We are using the techniques of $[15,17]$ to segment the cavities in the X-Ray images. Following diagram gives the rough idea of our work:

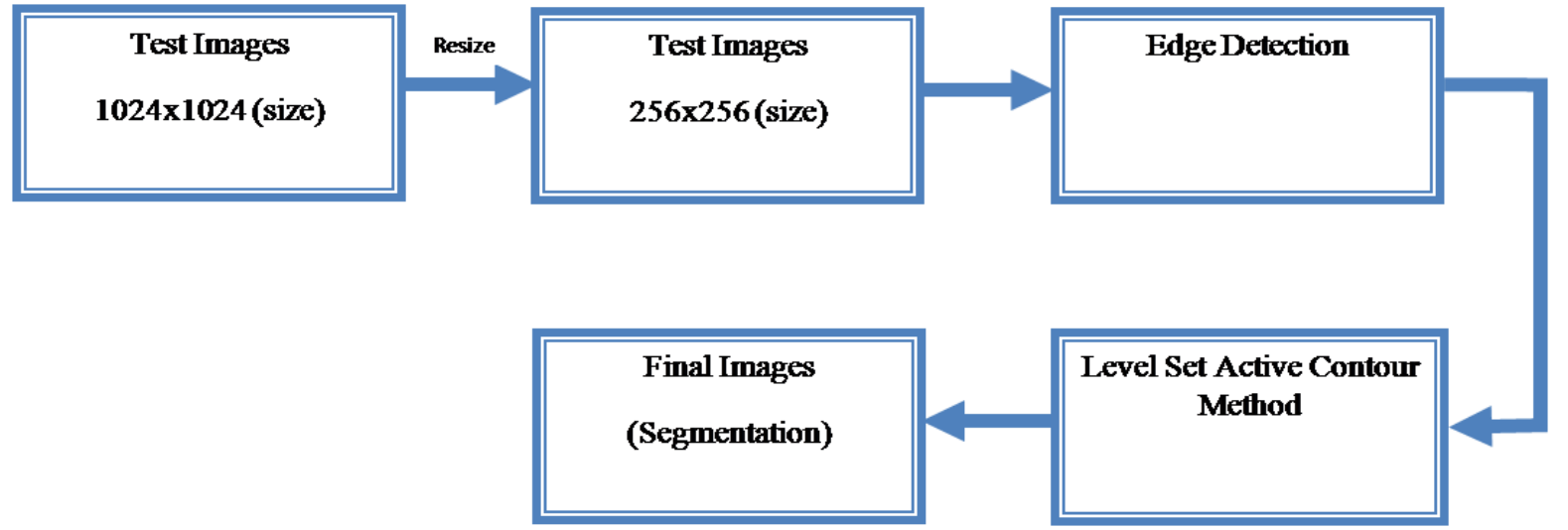

Figure 2: Flowchart of Methodology used

The proposed segmentation scheme segments the cavities present in the digital dental X-Ray images. The first step is the image enhancement step in which images are resized to 256x256. Further, images are converted from RGB color space to Gray Level color space. Next is the identification step in which the cavities are identified. After the Identification step, the edges are detected using any of the edge detection operators like sobel operator, canny operator, preywitt operator etc. Here we used sobel operator for edge detection. The Edge detection results in an image having edges spread over the boundaries of the cavities (REdge). The next step is LSAC method in which initial contours are set manually, near the object. After a number of iterations, the contours will take over the edges of the object and the region near to the object. The resultant is the segmented region which is Region of Interest (ROI).

The test images (ITest) are taken from a dental clinic of size $1024 \times 1024$ and following operations are performed:

Step1: The images are resized (IReseize) so as to get them fit to the screen, using image processing function for resizing the image.

Step2: The resultant image after step1 (IReseize), is then converted into gray scale image (IGray) because it becomes easy to analyze the image more easily if it is in gray scale [0 255].
Step3: In the edge detection process, the defects/cavities present in the image (IGray), are identified and the cavities are enhanced with covered edges (IEdge).

Step4: In LSAC, the cavities and the region near to the cavities are segmented by using Algorithm in [17]. The process for LSAC is:

- Set Initial Contours: Generate the initial regions or contours as rectangular region, and the co-ordinates are set manually, according to the cavities present in the image. For assistance, any other contour shape can also be used. Next we are using double well potential p2, which is good for both edge and region based models. The value of potential function decides, if the segmentation is based on edge based model or region based model or a combination of both the models.

- Level Set Evaluation (LSE): In LSE, the initial contours that are set manually on the image will automatically take over the object/cavities boundaries and over the region that may or may not be affected by the cavities.

Step5: Display segmented image. 


\section{RESULTS}

\subsection{Numerical Implementation}

In our method, the choice of parameters is easy and depends on the intensity of the cavities. Also, initial contours are set manually near to the cavity boundaries so that the segmentation time should be less. The level set evolution is implemented using DRLSE [17].

Some of the parameters used in our model are kept fixed such as time step

$(\Delta \mathrm{t})=1$ and $\mu=\frac{0.2}{\Delta t}$.

Other parameters are set as follows: lambda $(\lambda)=5$, sigma $(\sigma)$ $=0.8$, alpha $(\alpha)=-3$, epsilon $(\varepsilon)=1.5$. These parameters can be changed according to the user's assistance.

\subsection{Segmentation Results}

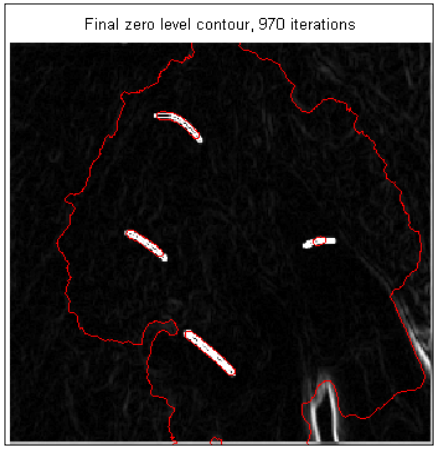

Figure 3.1 Final Zero Level Contour after 970 iterations

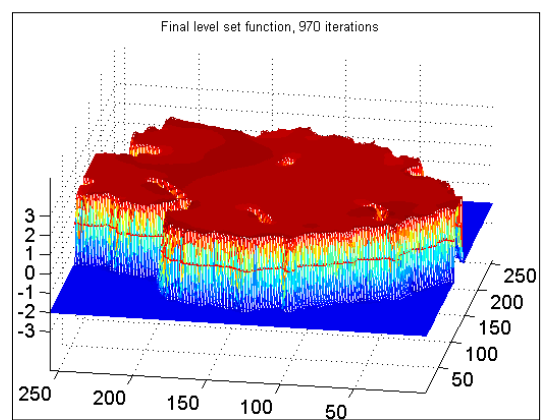

Figure 3.2 Final Level Set Function after 970 Iterations

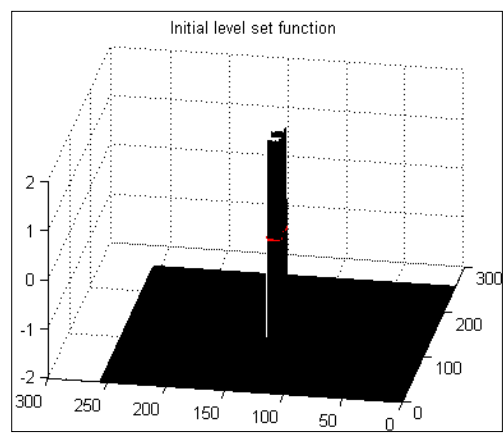

Figure 3.3 Initial Level Set Function

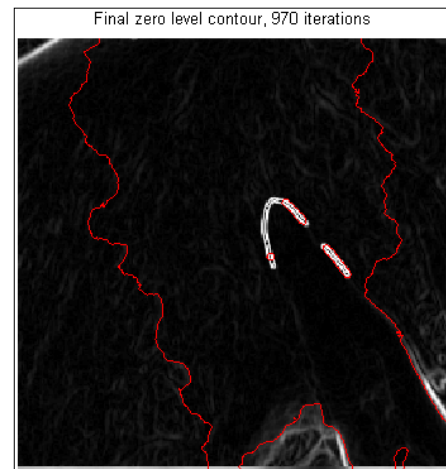

Figure 4.1 Final Zero Level Contour after 970 iterations

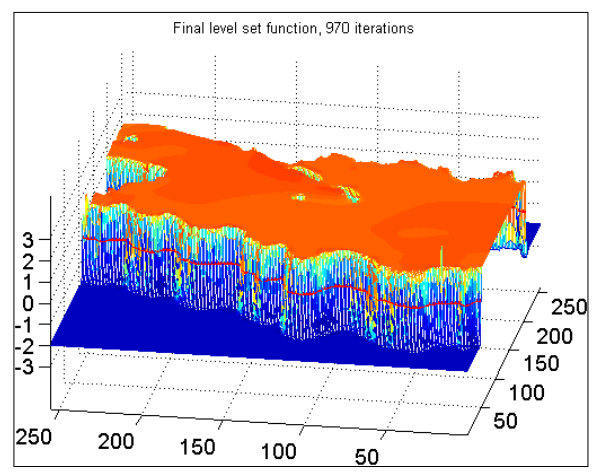

Figure 4.2 Final Level Set Function after 970 Iterations

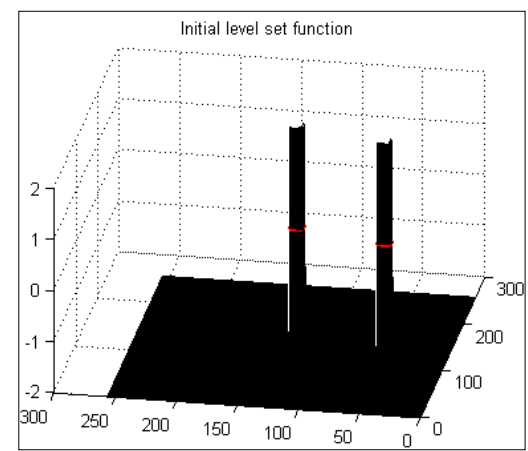

Figure 4.3 Initial Level Set Function

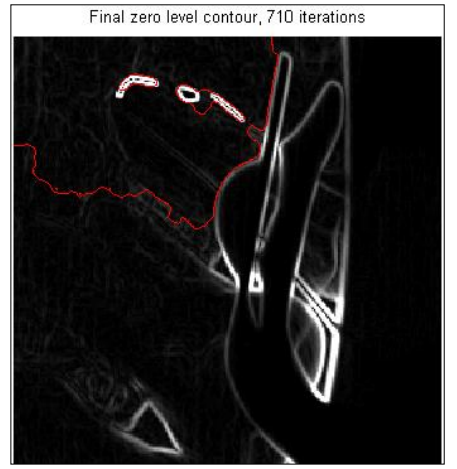

Figure 5.1 Final Zero Level Contour after 710 iterations 


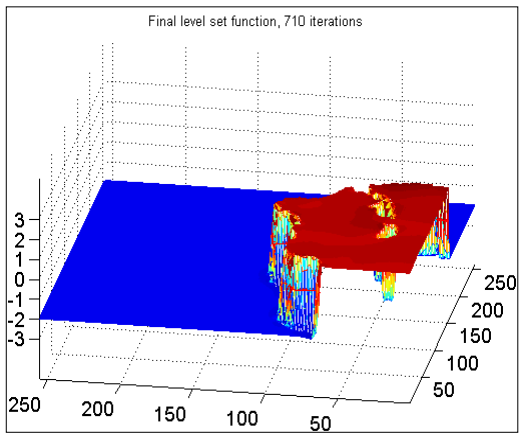

Figure 5.2 Final Level Set Function after 710 Iterations

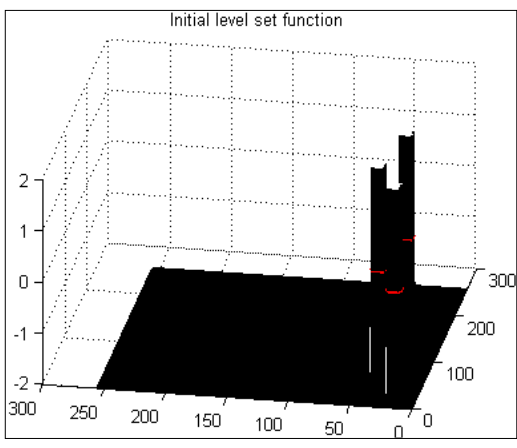

Figure 5.3 Initial Level Set Function

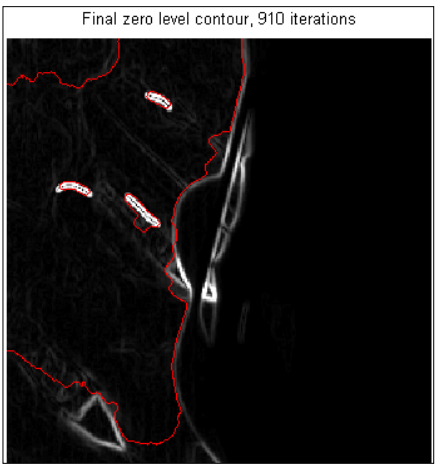

Figure 6.1 Final Zero Level Contour after 910 iterations

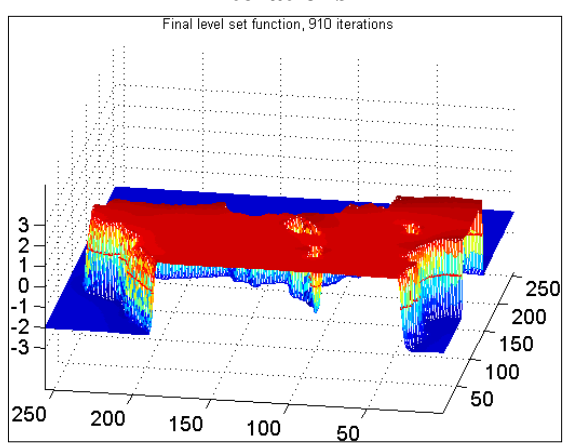

Figure 6.2 Final Level Set Function after 910 Iterations

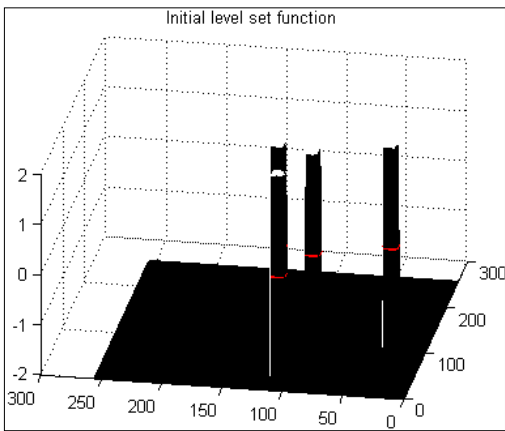

Figure 6.3 Initial Level Set Function

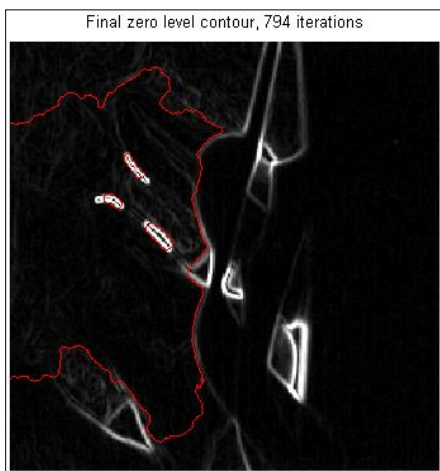

Figure 7.1 Final Zero Level Contour after 794 iterations

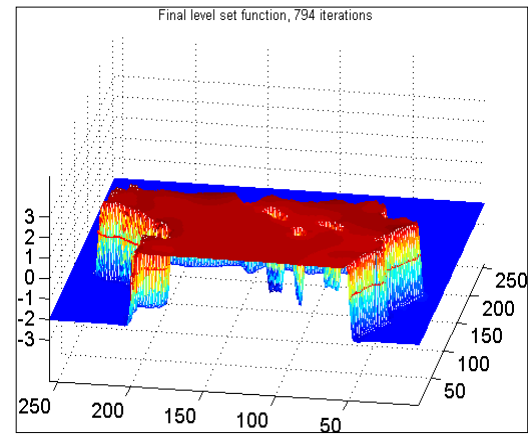

Figure 7.2 Final Level Set Function after 794

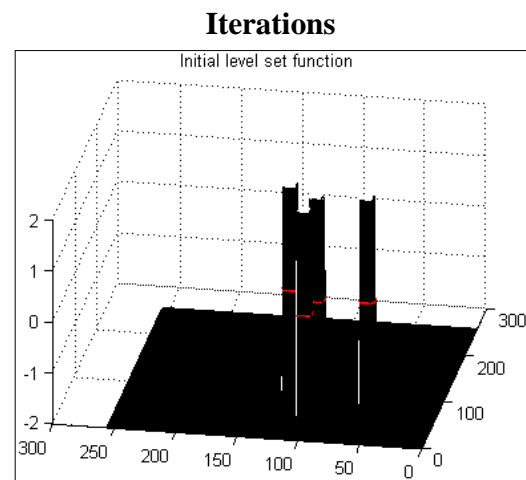

Figure 7.3 Initial Level Set Function.

\section{CONCLUSION}

The images are resized so as to reduce the time complexity of the Level Set Active Contour method as well as the errors (if any). Greater will be the size of the image, greater will be the time taken by the contours to spread over the boundaries. As the size of the image is decreases, the time complexity is 
reduced. The performance of the algorithm is tested with different sizes i.e. 1024 x 1024, 512 x 512, 256 x 256, 64 x 64 , of the images and accordingly the size of the image is chosen. We have chosen images with size 512 x 512 because with sizes $64 \times 64$ and $256 \times 256$, the images become very small that the cavities/defects cannot be identified properly. Also, with the size $1024 \times 1024$, the images become too large that it does not fit to the screen.

\section{REFERENCES}

[1] M. A.Balafar, ABD.Rahman Ramli, M.Iqbal Saripan, Rozi.Mahmud, Syamsiah Mashohor, Molod. Balafar "New Multi-scale Medical Image Segmentation based on Fuzzy CMean (FCM)" IEEE Conference on Innovative Technologies in Intelligent Systems and Industrial Applications (CITISIA), pp 66-70, ISBN: 978-1-42442416-0, 2008

[2] N. Senthilkumaran, "Edge Detection for Dental X-ray Image Segmentation using Neural Network approach", The International Journal of Computer Science \& Applications (TIJCSA) Volume 1, No. 7, September 2012 ISSN - 2278-1080, 2012

[3] M. Kass, A. Witkin and D. Terzopoulos, "Snakes: Active Contour Models," International Journal of Computer Vision, 1(4): 321-331,1988.

[4] V. Caselles, R. Kimmel, and G. Sapiro, "Geodesic active contours," in IJCV, 1997.

[5] Xu, J. L. Prince, "Gradient Vector Flow: A New External Force for Snakes," IEEE Proceedings on Computer Vision and Pattern Recognition, 66-71,1997.

[6] Steve R. Gunn, Mark S. Nixon, "A Robust Snake Implementation; A Dual Active Contour" , IEEE Transactions on Pattern Analysis and Machine Intelligence, VOL. 19, NO. 1, 1997

[7] J. A. Sethian, "Level Set Methods and Fast Marching Methods," Evolving Interfaces in Computational Geometry, Fluid Mechanics, Computer Vision and Materials Science, 1st. ed. Cambridge University Press, 1999.

[8] G. A. Giraldi, E. Strauss and A. A. F. Oliveira, "A Boundary Extraction Approach Based on MultiResolution Methods and the T-Snakes Framework," DEL, UFRJ,2000.
[9] T. Chan and L. Vese, "Active contours without edges," in IEEE Trans. on Image Processing, 2001.

[10] Foued Derraz, Mohamed Beladgham, M'hamed Khelif, "Application of Active Contour Models in Medical Image Segmentation", Information Technology: Coding and Computing (ITCC), pp 675 - 681 Vol.2, ISBN: 07695-2108-8, April 2004

[11] Li, C. Xu, C. Gui, et al, "Level set evolution without reinitialization: A new variational formulation," in Proc. IEEE Conf. Computer Vision and Pattern Recognition(CVPR), San Diego, 2005, vol. 1, pp. 430436, 2005

[12] Xujia Qin, Suqiong Zhang, "New Medical Image Sequences Segmentation Based On Level Set Method", Image Analysis And Signal Processing (IASP), Pp 22 27, ISBN: 978-1-4244-3987-4, April 2009

[13] Yun-Jen Chiu, Van-Truong Pham, Thi-Thao Tran, KuoKai Shyu, "Evaluation of Active Contour on Medical Inhomogeneous Image Segmentation", Computer Science and Information Technology (ICCSIT), pp 311 314, ISBN: 978-1-4244-5537-9, July 2010

[14] Wei Yu, Franz Franchetti, Yao-Jen Chang, Tsuhan Chen, "Fast And Robust Active Contours For Image Segmentation", Image Processing (ICIP), pp 641 - 644, ISBN: 978-1-4244-7992-4, Sept. 2010

[15] Chunming Li, Rui Huang, Zhaohua Ding, J. Chris Gatenby, Dimitris N. Metaxas, John C. Gore, "A Level Set Method For Image Segmentation In The Presence Of Intensity Inhomogeneities With Application To MRI", IEEE Transactions on Image Processing, pp 2007-2016 VOL. 20, July 2011

[16] Abdolvahab Ehsani Rad, Mohd Shafry Mohd Rahim, Alireza Norouzi, "Digital Dental X-Ray Image Segmentation And Feature Extraction”, TELKOMNIKA, Pp. 3109 - 3114, E-ISSN: 2087-278X, June 2013

[17] Chunming Li, Chenyang Xu, Changfeng Gui, Martin D. Fox, "Distance Regularized Level Set Evolution And Its Application To Image Segmentation", IEEE Transactions on Image Processing, VOL. 19, Pp 3243-3253, December 2010 